\title{
Spike timing and the coding of naturalistic sounds in a central auditory area of songbirds
}

\author{
Brian D. Wright, ${ }^{1-3}$ Kamal Sen, ${ }^{1-3}$ William Bialek ${ }^{1,4,5}$ and Allison J. Doupe ${ }^{1-3}$ \\ ${ }^{1}$ Sloan-Swartz Center for Theoretical Neurobiology \\ ${ }^{2}$ Departments of Physiology and ${ }^{3}$ Psychiatry \\ University of California at San Francisco, San Francisco, California 94143-0444 \\ ${ }^{4}$ NEC Research Institute, 4 Independence Way, Princeton, New Jersey 08540 \\ ${ }^{5}$ Department of Physics, Princeton University, Princeton, New Jersey 08544 \\ $\{$ bdwright/kamal/ajd\}@phy.ucsf.edu,wbialek@princeton.edu
}

January 12, 2002

\begin{abstract}
In nature, animals encounter high dimensional sensory stimuli that have complex statistical and dynamical structure. Attempts to study the neural coding of these natural signals face challenges both in the selection of the signal ensemble and in the analysis of the resulting neural responses. For zebra finches, naturalistic stimuli can be defined as sounds that they encounter in a colony of conspecific birds. We assembled an ensemble of these sounds by recording groups of 10-40 zebra finches, and then analyzed the response of single neurons in the songbird central auditory area (field L) to continuous playback of long segments from this ensemble. Following methods developed in the fly visual system, we measured the information that spike trains provide about the acoustic stimulus without any assumptions about which features of the stimulus are relevant. Preliminary results indicate that large amounts of information are carried by spike timing, with roughly half of the information accessible only at time resolutions better than $10 \mathrm{~ms}$; additional information is still being revealed as time resolution is improved to $2 \mathrm{~ms}$. Information can be decomposed into that carried by the locking of individual spikes to the stimulus (or modulations of spike rate) vs. that carried by timing in spike patterns. Initial results show that in field L, temporal patterns give at least $\sim 20 \%$ extra information. Thus, single central auditory neurons can provide an informative representation of naturalistic sounds, in which spike timing may play a significant role.
\end{abstract}

\section{Introduction}

Nearly fi fty years ago, Barlow []] and Attneave [3] suggested that the brain may construct a neural code that provides an effi cient representation for the sensory stimuli that occur in the natural world. Slightly earlier, MacKay and McCulloch [3] emphasized that neurons that could make use of spike timingrather than a coarser "rate code"-would have available a vastly larger capacity to convey information, although they left open the question of whether this capacity is used effi ciently. Theories for timing codes and effi cient representation have been discussed extensively, but the evidence for these attractive ideas 
remains tenuous. A real attack on these issues requires (at least) that we actually measure the information content and effi ciency of the neural code under stimulus conditions that approximate the natural ones. In practice, constructing an ensemble of "natural" stimuli inevitably involves compromises, and the responses to such complex dynamic signals can be very diffi cult to analyze.

At present the clearest evidence on effi ciency and timing in the coding of naturalistic stimuli comes from central invertebrate neurons [4, 5] and from the sensory periphery [6, 7] and thalamus [8, 9] of vertebrates. The situation for central vertebrate brain areas is much less clear. Here we use the songbird auditory system as an accessible test case for these ideas. The set of songbird telencephalic auditory areas known as the fi eld L complex is analogous to mammalian auditory cortex and contains neurons that are strongly driven by natural sounds, including the songs of birds of the same species (conspecifi cs) 10, 11, 12, 13. We record from the zebra fi nch fi eld L, using naturalistic stimuli that consist of recordings from groups of 10-40 conspecific birds. We fi nd that single neurons in fi eld L show robust and well modulated responses to playback of long segments from this song ensemble, and that we are able to maintain recordings of suffi cient stability to collect the large data sets that are required for a model independent information theoretic analysis. Here we give a preliminary account of our experiments.

\section{A naturalistic ensemble}

Auditory processing of complex sounds is critical for perception and communication in many species, including humans, but surprisingly little is known about how high level brain areas accomplish this task. Songbirds provide a useful model for tackling this issue, because each bird within a species produces a complex individualized acoustic signal known as a song, which reflects some innate information about the species' song as well as information learned from a "tutor" in early life. In addition to learning their own song, birds use the acoustic information in songs of others to identify mates and group members, to discriminate neighbors from intruders, and to control their living space [14]. Consistent with how ethologically critical these functions are, songbirds have a large number of forebrain auditory areas with strong and increasingly specialized responses to songs [11, 15, 16]. The combination of a rich set of behaviorally relevant stimuli and a series of high-level auditory areas responsive to those sounds provides an opportunity to reveal general principles of central neural encoding of complex sensory stimuli. Many prior studies have chosen to study neural responses to individual songs or altered versions thereof. In order to make the sounds studied increasingly complex and natural, we have made recordings of the sounds encountered by birds in our colony of zebra fi nches. To generate the sound ensemble that was used in this study we fi rst created long records of the vocalizations of groups of 10-40 zebra fi nches in a soundproof acoustic chamber with a directional microphone above the bird cages. The group of birds generated a wide variety of vocalizations including songs and a variety of different types of calls. Segments of these sounds were then joined to create the sounds presented in the experiment. One of the segments that was presented $(\sim 30 \mathrm{sec})$ was repeated in alternation with different segments.

We recorded the neural responses in fi eld $\mathrm{L}$ of one of the birds from the group to the ensemble of natural sounds played back through a speaker, at an intensity approximately equal to that in the colony recording. This bird was lightly anesthetized with urethane. We used a single electrode to record the neural response waveforms and sorted single units offline. Further details concerning experimental techniques can be found in Ref. [13]. 
A

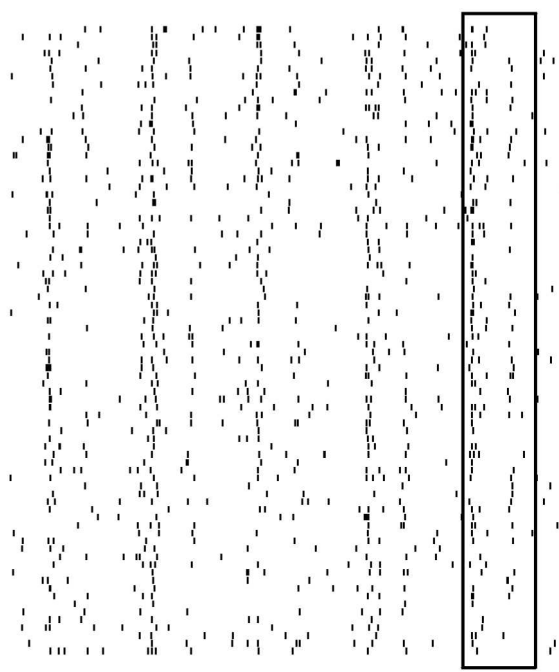

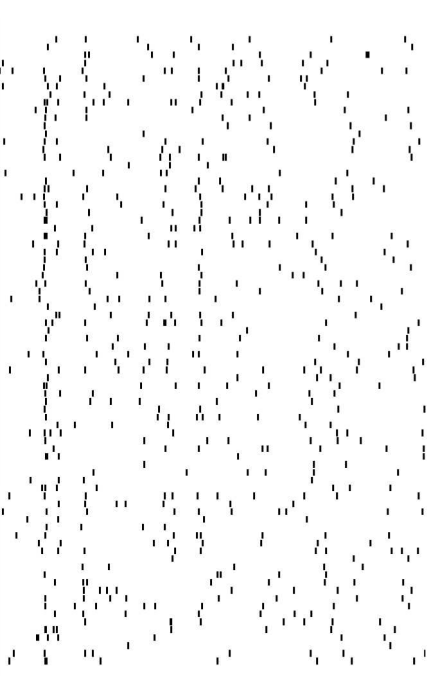

$500 \mathrm{~ms}$
D

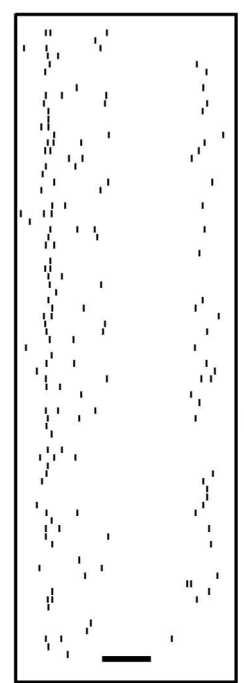

B

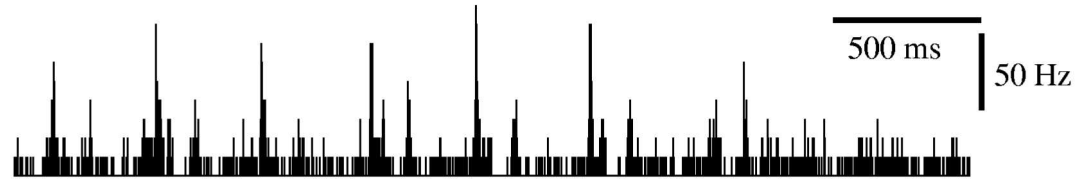

C

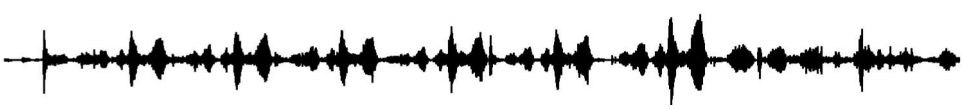

Figure 1: A. Spike raster of 4 seconds of the responses of a single neuron in fi eld $\mathrm{L}$ to a 30 second segment of a natural sound ensemble of zebra fi nch sounds. The stimulus was repeated 80 times. B. Peri-stimulus time histogram (PSTH) with $1 \mathrm{~ms}$ bins. C. Sound pressure waveform for the natural sound ensemble. D. Blowup of segment shown in the box in A. The scale bar is $50 \mathrm{~ms}$.

\section{Information in spike sequences}

The auditory telencephalon of birds consists of a set of areas known as the fi eld $\mathrm{L}$ complex, which receive input from the auditory thalamus and project to increasingly selective auditory areas such as NCM, cHV and NIf [12, 17] and ultimately to the brain areas specialized for the bird's own song. Field L neurons respond to simple stimuli such as tone bursts, and are organized in a roughly tonotopic fashion [18], but also respond robustly to many complex sounds, including songs. Figure 1 shows 4 seconds of the responses of a cell in fi eld $\mathrm{L}$ to repeated presentations of a $30 \mathrm{sec}$ segment from the natural ensemble described above. Averaging over presentations, we see that spike rates are well modulated. Looking at the responses on a fi ner time resolution we see that aspects of the spike train are reproducible on at least $\mathrm{a} \sim 10 \mathrm{~ms}$ time scale. This encourages us to measure the information content of these responses over a range of time scales, down to millisecond resolution.

Our approach to estimating the information content of spike trains follows Ref. [他. At some time $t$ (defi ned relative to the repeating stimulus) we open a window of size $T$ to look at the response. Within this window we discretize the spike arrival times with resolution $\Delta \tau$ so that the response becomes a 


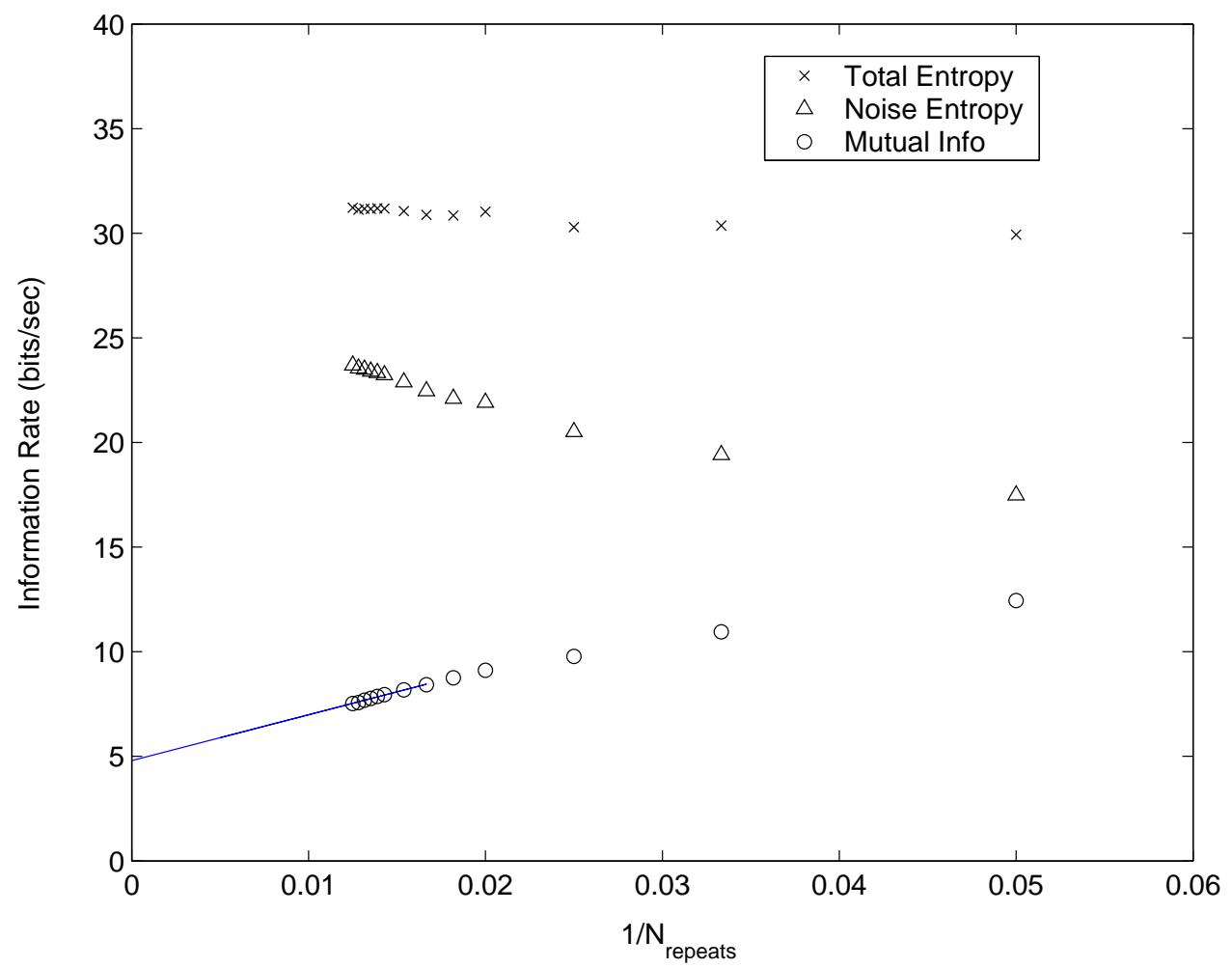

Figure 2: Mutual information rate for the spike train is shown as a function of data size for $\Delta \tau=2 \mathrm{~ms}$ and $T=32 \mathrm{~ms}$.

"word" with $T / \Delta \tau$ letters. If the time resolution $\Delta \tau$ is very small, the allowed letters are only 1 and 0 , but as $\Delta \tau$ becomes larger one must keep track of multiple spikes within each bin. Examining the whole experiment, we sample the probability distribution of words, $P_{T}(W)$, and the entropy of this distribution sets the capacity of the code to convey information about the stimulus:

$$
S_{\text {total }}(T ; \Delta \tau)=-\sum_{W} P_{T}(W) \log _{2} P_{T}(W) \text { bits },
$$

where the notation reminds us that the entropy depends both on the size of the words that we consider and on the time resolution with which we classify the responses. We can think of this entropy as measuring the size of the neuron's vocabulary.

Because the whole experiment contributes to defi ning the vocabulary size, estimating the distribution $P_{T}(W)$ and hence the total entropy is not signifi cantly limited by the problems of fi nite sample size. This can be seen in Fig. 2 in the stability of the total entropy with changing the number of repeats used in the analysis. Here we show the total entropy as a rate in bits per second by dividing the entropy by the time window $T$.

While the capacity of the code is limited by the total entropy, to convey information particular words in the vocabulary must be associated, more or less reliably, with particular stimulus features. If we look 
at one time $t$ relative to the (long) stimulus, and examine the words generated on repeated presentations, we sample the conditional distribution $P_{T}(W \mid t)$. This distribution has an entropy that quantifi es the noise in the response at time $t$, and averaging over all times we obtain the average noise entropy,

$$
S_{\text {noise }}(T ; \Delta \tau)=\left\langle-\sum_{W} P_{T}(W \mid t) \log _{2} P_{T}(W \mid t)\right\rangle_{t} \text { bits, }
$$

where $\langle\cdots\rangle_{t}$ indicates a time average (in general, $\langle\cdots\rangle_{x}$ denotes an average over the variable $x$ ). Technically, the above average should be an average over stimuli $s$, however, for a suffi ciently long and rich stimulus, the ensemble average over $s$ can be replaced by a time average. For the noise entropy, the problem of sampling is much more severe, since each distribution $P_{T}(W \mid t)$ is estimated from a number of examples given by the number of repeats. Still, as shown in Fig. 2, we fi nd that the dependence of our estimate on sample size is simple and regular; specifi cally, we fi nd

$$
S\left(T ; \Delta \tau ; N_{\text {repeats }}\right)=S(T ; \Delta \tau ; \infty)+\frac{A}{N_{\text {repeats }}}+\cdots .
$$

This is what we expect for any entropy estimate if the distribution is well sampled, and if we make stronger assumptions about the sampling process (independence of trials etc.) we can even estimate the correction coeffi cient $A[1 \phi]$. In systems where much larger data sets are available this extrapolation procedure has been checked, and the observation of a good fit to Eq. 目) is a strong indication that larger sample sizes will be consistent with $S(T ; \Delta \tau)=S(T ; \Delta \tau ; \infty)$; further, this extrapolation can be tested against bounds on the entropy that are derived from more robust quantities [ [ 4 . Most importantly, failure to observe Eq. (3) means that we are in a regime where sampling is not suffi cient to draw reliable conclusions without more sophisticated arguments, and we exclude these regions of $T$ and $\Delta \tau$ from our discussion.

Ideally, to measure the spike train total and noise entropy rates, we want to go to the limit of infi nite word duration. A true entropy is extensive, which here means that it grows linearly with spike train word duration $T$, so that the entropy rate $\mathcal{S}=S / T$ is constant. For fi nite word duration however, words sampled at neighboring times will have correlations between them due, in part, to correlations in the stimulus (for birdsong these stimulus autocorrelation time scales can extend up to $\sim 100 \mathrm{~ms}$ ). Since the word samples are not completely independent, the raw entropy rate is an overestimate of the true entropy rate. The effect is larger for smaller word duration and the leading dependence of the raw estimate is

$$
\mathcal{S}(T ; \Delta \tau ; \infty)=\mathcal{S}(\infty ; \Delta \tau ; \infty)+\frac{B}{T}+\cdots,
$$

where $B>0$ and we have already taken the infi nite data size limit. We cannot directly take the large $T$ limit, since for large word lengths we eventually reach a data sampling limit beyond which we are unable to reliably compute the word distributions. On the other hand, if there is a range of $T$ for which the distributions are suffi ciently well sampled, the behavior in Eq. (4) should be observed and can be used to extrapolate to infi nite word size [4]. We have checked that our data shows this behavior and that it sets in for word sizes below the limit where the data sampling problem occurs. For example, in the case of the noise entropy, for $\Delta \tau=2 \mathrm{~ms}$, it applies for $T$ below the limit of $50 \mathrm{~ms}$ (above this we run into sampling problems). The total entropy estimate is nearly perfectly extensive.

Finally, we combine estimates of total and noise entropies to obtain the information that words carry about the sensory stimulus,

$$
I(T ; \Delta \tau)=S_{\text {total }}(T ; \Delta \tau)-S_{\text {noise }}(T ; \Delta \tau) \text { bits. }
$$


Figure 2 shows the total and noise entropy rates as well as the mutual information rate for a time window $T=32 \mathrm{~ms}$ and time resolution $\Delta \tau=2 \mathrm{~ms}$. The error bars on the raw entropy and information rates were estimated to be approximately \pm 0.2 bits/sec using a simple bootstrap procedure over the repeated trials. The extrapolation to infi nite data size is shown for the mutual information rate estimate (error bars in the extrapolated values will be $< \pm 0.2 \mathrm{bits} / \mathrm{sec}$ ) and is consistent with the prediction of Eq. (3). Since the total entropy is nearly extensive and the noise entropy rate decreases with word duration due to subextensive corrections as described above, the mutual information rate shown in Fig. 2 grows with word duration. We find that there is an upward change in the mutual information rate (computed at $\Delta \tau=2 \mathrm{~ms}$ and $T=32 \mathrm{~ms}$ ) of $\sim 7 \%$, in the large $T$ limit. For simplicity in the following, we shall look at a fi xed word duration $T=32 \mathrm{~ms}$ that is in the well-sampled region for all time resolutions $\Delta \tau$ considered.

The mutual information rate measures the rate at which the spike train removes uncertainty about the stimulus. However, the mutual information estimate does not depend on identifying either the relevant features of the stimulus or the relevant features of the response, which is crucial in analyzing the response to such complex stimuli. In this sense, our estimates of information transmission and effi ciency are independent of any model for the code, and provide a benchmark against which such models could be tested.

One way to look at the information results is to fi $\mathrm{x}$ our time window $T$ and ask what happens as we change our time resolution $\Delta \tau$. When $\Delta \tau=T$, the "word" describing the response is nothing but the number of spikes in the window, so we have a rate or counting code. As we decrease $\Delta \tau$, we gradually distinguish more and more detail in the arrangement of spikes in the window. We chose a range of $T$ values from $30-100 \mathrm{~ms}$ in our analyses to cover previously observed response windows for fi eld $\mathrm{L}$ neurons and to probe the behaviorally relevant time scale $(\sim 100 \mathrm{~ms})$ of individual song syllables or notes. For $T=32 \mathrm{~ms}$, we show the results (extrapolated to infi nite data size) in the upper curve of Fig. 3. The spike train mutual information shows a clear increase as the timing resolution is improved. In addition, Fig. 3 shows that roughly half of the information is accessible at time resolutions better than $10 \mathrm{~ms}$ and additional information is still being revealed as time resolution is improved to $2 \mathrm{~ms}$.

\section{Information in rate modulation}

Knowing the mutual information between the stimulus and the spike train (defi ned in the window $T$ ), we would like to ask whether this can be accounted for by the information in single spike events or whether there is some additional information conveyed by the patterns of spikes. In the latter case, we have precisely what we mean by a temporal or timing code: there is information beyond that attributable to the probability of single spike events occurring at time $t$ relative to the onset of the stimulus. By event at time $t$, we mean that the event occurs between time $t$ and time $t+\Delta \tau$, where $\Delta \tau$ is the resolution at which we are looking at the spike train. This probability is simply proportional to the fi ring rate (or peri-stimulus time histogram (PSTH)) $r(t)$ at time $t$ normalized by the mean fi ring rate $\bar{r}$. Specifi cally if the duration of each repeated trial is $T_{\text {repeat }}$ we have

$$
P\left(1 \text { spk @ } t \mid s\left(t^{\prime}\right)\right)=\frac{r(t) \Delta \tau}{\bar{r} T_{\text {repeat }}},
$$

where $s\left(t^{\prime}\right)$ denotes the stimulus history $\left(t^{\prime}<t\right)$. The probability of a spike event at $t$, a priori of knowing the stimulus history, is flat: $P(1 \mathrm{spk} @ t)=\Delta \tau / T_{\text {repeat }}$. Thus, the mutual information between the 


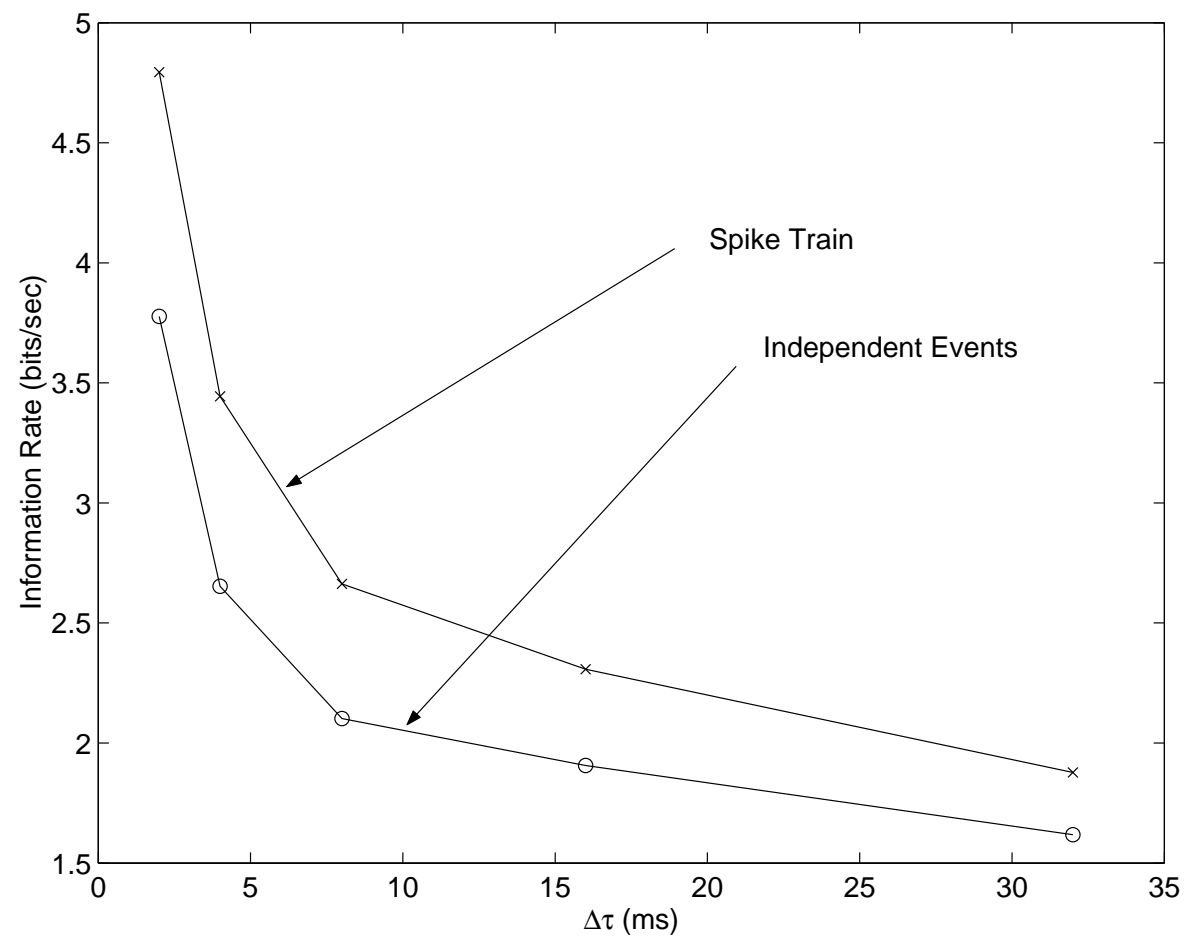

Figure 3: Information rates for the spike train $(T=32 \mathrm{~ms})$ and single spike events as a function of time resolution $\Delta \tau$ of the spike rasters, corrected for fi nite data size effects.

stimulus and the single spike events is [20]:

$$
\begin{aligned}
I(1 \text { spike; } \Delta \tau) & =S[P(1 \text { spk @ t })]-\langle S[P(1 \text { spk @ t } t s)]\rangle_{s} \\
& =\left\langle\frac{r(t)}{\bar{r}} \log _{2}\left(\frac{r(t)}{\bar{r}}\right)\right\rangle_{t} \text { bits, }
\end{aligned}
$$

where $r(t)$ is the PSTH binned to resolution $\Delta \tau$ and the stimulus average in the first expression is replaced by a time average in the second (as discussed in the calculation of the noise entropy in spike train words in the previous section). We find that this information is approximately 1 bit for $\Delta \tau=2$ ms. Supposing that the individual spike events are independent (i.e. no intrinsic spike train correlations), the information rate in single spike events is obtained by multiplying the mutual information per spike (Eq. 7) by the mean fi ring rate of the neuron $(\sim 3.5 \mathrm{~Hz})$. This gives an upper bound to the single spike event contribution to the information rate and is shown in the lower curve of Fig. 3 (error bars are again $< \pm 0.2 \mathrm{bits} / \mathrm{sec}$ ). Comparing with the spike train information (upper curve), we see that at a resolution of $\Delta \tau=2 \mathrm{~ms}$, there is at least $\sim 20 \%$ of the total information in the spike train that cannot be attributable to single spike events. Thus there is some pattern of spikes that is contributing synergistically to the mutual information. The fact discussed, in the previous section, that the spike train 
information rate grows subextensively with the the word duration out to the point where data sampling becomes problematic is further confi rmation of the synergy from spike patterns. Thus we have shown model-independent evidence for a temporal code in the neural responses.

\section{Conclusion}

Until now, few experiments on neural responses in high level, central vertebrate brain areas have measured the information that these responses provide about dynamic, naturalistic sensory signals. As emphasized in earlier work on invertebrate systems, information theoretic approaches have the advantage that they require no assumptions about the features of the stimulus to which neurons respond. Using this method in the songbird auditory forebrain, we found that patterns of spikes seem to be special events in the neural code of these neurons, since they carry more information than expected by adding up the contributions of individual spikes. It remains to be determined what these spike patterns are, what stimulus features they may encode, and what mechanisms may be responsible for reading such codes at even higher levels of processing.

\section{Acknowledgments}

Work at UCSF was supported by grants from the NIH (NS34835) and the Sloan-Swartz Center for Theoretical Neurobiology. BDW and KS supported by NRSA grants from the NIDCD. We thank Katrin Schenk and Robert Liu for useful discussions.

\section{References}

[1] Barlow, H.B. (1961). Possible principles underlying the transformation of sensory messages. In Sensory Communication, W.A. Rosenblith, ed., pp. 217-234 (MIT Press, Cambridge, MA).

[2] Attneave, F. (1954). Some informational aspects of visual perception. Psychol. Rev. 61, 183-193.

[3] MacKay, D. and McCulloch, W.S. (1952). The limiting information capacity of a neuronal link. Bull. Math. Biophys. 14, 127-135.

[4] Strong, S.P., Koberle, R., de Ruyter van Steveninck, R. and Bialek, W. (1998). Entropy and information in neural spike trains, Phys. Rev. Lett. 80, 197-200.

[5] Lewen, G.D., Bialek, W. and de Ruyter van Steveninck, R.R. (2001). Neural coding of naturalistic motion stimuli. Network 12, 317-329, physics/0103088.

[6] Rieke, F., Bodnar, D.A. and Bialek, W. (1995). Naturalistic stimuli increase the rate and effi ciency of information transmission by primary auditory afferents. Proc. R. Soc. Lond. B 262, 259-265.

[7] Berry II, M.J., Warland, D.K. and Meister, M. (1997). The structure and precision of retinal spike trains. Proc. Nat. Acad. Sci. (USA) 94, 5411-5416.

[8] Reinagel, P. and Reid, R.C. (2000). Temporal coding of visual information in the thalamus. J. Neurosci. 20, 5392-5400. 
[9] Liu, R.C., Tzonev, S., Rebrik, S. and Miller, K.D. (2001). Variability and information in a neural code of the cat lateral geniculate nucleus. J. Neurophysiol. 86, 2789-2806.

[10] Scheich, H., Langner, G. and Bonke, D. (1979). Responsiveness of units in the auditory neostriatum of the guinea fowl (Numida meleagris) to species-specifi c calls and synthetic stimuli II. Discrimination of Iambus-Like Calls. J. Comp. Physiol. A 132, 257-276.

[11] Lewicki, M.S. and Arthur, B.J. (1996). Hierarchical organization of auditory temporal context sensitivity. J. Neurosci. 16(21), 6987-6998.

[12] Janata, P. and Margoliash, D. (1999). Gradual emergence of song selectivity in sensorimotor structures of the male zebra fi nch song system. J. Neurosci. 19(12), 5108-5118.

[13] Theunissen, F.E., Sen, K. and Doupe, A.J. (2000). Spectral temporal receptive fi elds of nonlinear auditory neurons obtained using natural sounds. J. Neurosci. 20(6), 2315-2331.

[14] Searcy, W.A. and Nowicki, S. (1999). In The Design of Animal Communication, M.D. Hauser and M. Konishi, eds., pp. 577-595 (MIT Press, Cambridge, MA).

[15] Margoliash, D. (1983). Acoustic parameters underlying the responses of song-specifi c neurons in the white-crowned sparrow. J. Neurosci. 3(5), 1039-1057.

[16] Sen, K., Theunissen, F.E. and Doupe, A.J. (2001). Feature analysis of natural sounds in the songbird auditory forebrain. J. Neurophysiol. 86, 1445-1458.

[17] Stripling, R., Kruse, A.A. and Clayton, D.F. (2001). Development of song responses in the zebra fi nch caudomedial neostriatum: role of genomic and electrophysiological activities. J. Neurobiol. 48, $163-180$.

[18] Zaretsky, M.D. and Konishi, M. (1976). Tonotopic organization in the avian telencephalon. Brain Res. 111, 167-171.

[19] Treves, A. and Panzeri, S. (1995). The upward bias in measures of information derived from limited data samples. Neural Comput., 7, 399-407.

[20] Brenner, N., Strong, S., Koberle, R. and Bialek, W. (2000). Synergy in a neural code, Neural Comput. 12, 1531-1552, physics/9902067. 